EPJ Web of Conferences 73, 04001 (2014)

DOI: $10.1051 /$ epjconf/20147304001

(C) Owned by the authors, published by EDP Sciences, 2014

\title{
Dynamical understanding of baryon resonances
}

\author{
Toru Sato \\ Department of Physics, Osaka University, Japan
}

\begin{abstract}
The nucleon resonances are investigated within a dynamical coupled-channels model of $\pi N$ and $\gamma N$ reactions up to the invariant mass $W=2 \mathrm{GeV}$. The pole positions and the $N^{*} \rightarrow \pi N, \gamma N, \eta N, K \Lambda, K \Sigma$ transition amplitudes at resonance poles are extracted from the meson-baryon partial wave amplitudes.
\end{abstract}

\section{Introduction}

Properties of the excited state of nucleons $N^{*}$ are extracted from the $\pi N$ and electromgnetic meson production reactions. In the region of $N^{*}$ mass below $2 \mathrm{GeV}$, the main reaction channels are $\pi N, \eta N, K \Lambda, K \Sigma, \omega N$ and $\pi \pi N$. Extraction of the resonance properties needs systematic account of those reactions [1-3].

In this paper, we briefly report on our first simultaneous analysis of pion and photon induced meson production reactions within the dynamical coupled channel(DCC) model [4]. The meson-baryon $(M B)$ channels included in the calculations are $M B=\pi N, \eta N, K \Lambda, K \Sigma$, and $\pi \pi N$ that has $\pi \Delta, \rho N$, and $\sigma N$ as doorway states. The meson-baryon amplitudes are calculated from solving a set of coupled-channels integral equations defined by an interaction Hamiltonian consisting of meson-exchange interactions and vertex interactions for describing the transition of a bare excited nucleon state $N^{*}$ to a meson-baryon channel $M B$ [1]. Brief description of the DCC model and the analysis of the meson production reactions is given in Sect. 2.

The pole positions and residues of nucleon resonances are extracted by analytically continuing the meson-baryon amplitudes. We compare the resonance parameters from our analysis with those given by the Particle Data Group [5] and the recent coupled-channels analyses by the Jülich [6] and BonnGatchina groups [7] in Sect. 3.

\section{Dynamical model of meson production reaction}

We start from the effective Hamiltonian which describes the interactions among the nucleon excited states(bare $N^{*}$ ), meson-baryon and $\pi \pi N$. By eliminating the one-body $N^{*}$ and three-body $\pi \pi N$ Fockspace components, we arrive at the following coupled channel equation for angular momentum $(J)$,

\footnotetext{
ae-mail: tsato@phys.sci.osaka-u.ac.jp
}

This is an Open Access article distributed under the terms of the Creative Commons Attribution License 4.0, which permits unrestricted use, distribution, and reproduction in any medium, provided the original work is properly cited. 

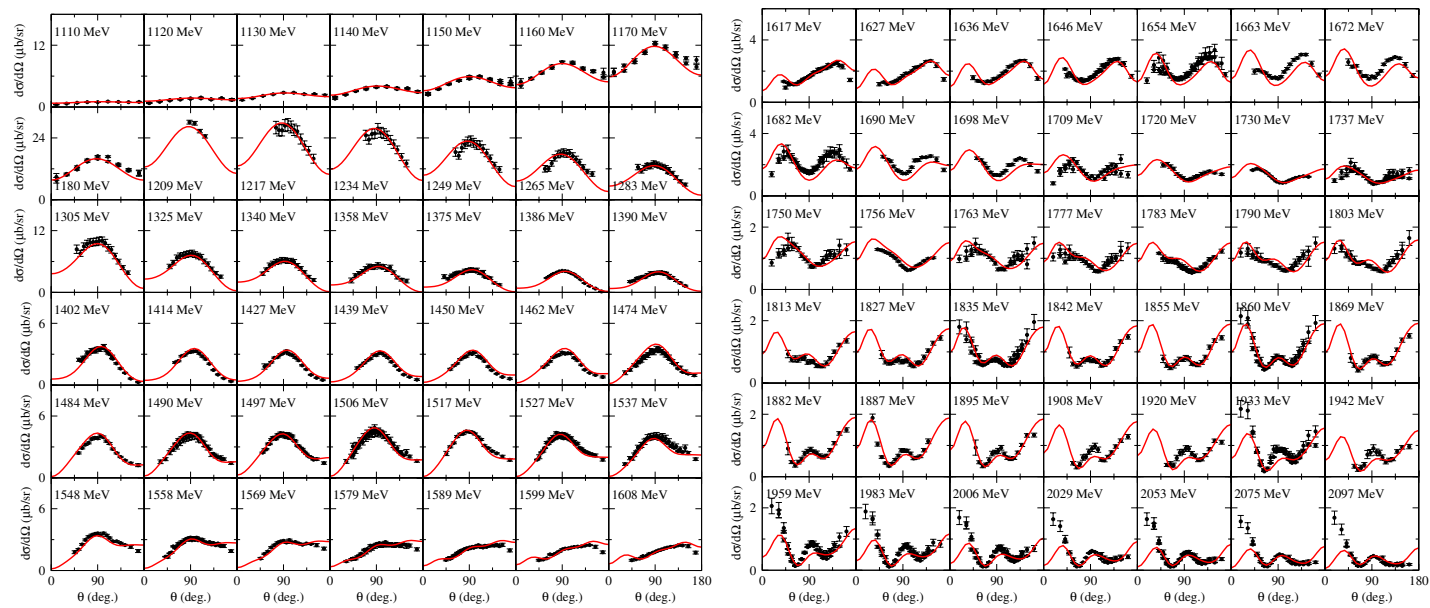

Figure 1. $d \sigma / d \Omega$ of $\gamma p \rightarrow \pi^{0} p$.

isospin $(I)$, parity $(P)$ state

$$
T_{\beta, \alpha}^{I J P}\left(k^{\prime}, k, W\right)=V_{\beta, \alpha}^{I J P}\left(k^{\prime}, k, W\right)+\sum_{\gamma} \int d q q^{2} V_{\beta, \alpha}^{I J P}\left(k^{\prime}, q, W\right) G_{\gamma}^{0}(q, W) T_{\gamma, \alpha}^{I J P}(q, k, W) .
$$

Here $\alpha, \beta$ are two-body meson-baryon states of stable particles $\pi N, \eta N, K \Lambda, K \Sigma$ and two-particle states with unstable particle $\rho N, \sigma N, \pi \Delta$ as doorway states of $\pi \pi N$ channel. The effective interaction $V_{\beta, \alpha}^{I J P}\left(k^{\prime}, k, W\right)$ includes non-resonant interactions for meson-baryon states, pole terms of bare $N^{*}$ and the Z-diagram with intermediate $\pi \pi N$ states.

The model parameters are determined by fitting the data of pion and photon induced meson production reactions off a proton. Our main challenge is to determine $N^{*}$ bare mass, meson-baryon coupling constants and vertex function. We take the following strategy. We first adjust the parameters associated with the non-resonant meson-baryon interaction to fit the $\pi N$ partial wave amplitude at low energy region $W<1.4 \mathrm{GeV}$. Then we extend the fits of the $\pi N$ amplitude up to $W=2.3 \mathrm{GeV}$ by including least number of $N^{*}$ states. After completing fit of the $\pi N$ amplitudes, we include the inelastic $\pi N$ reaction and then include the photon induced reactions by tuning the model parameters. The data included in this analysis and the description of the meson production cross sections and polarization observables are given in Ref. [4].

Here, we show one example of the differential cross section of $\gamma p \rightarrow \pi^{0} p$ in Fig. 1. In our previous analysis, we only adjusted the photo coupling of bare $N^{*}$ and only data below $W<1.6 \mathrm{GeV}$ were fitted well. In this work, all of the model parameters are varied to fit simultaneously the data of pion and photon induced reactions. This change of strategy gives us more freedom to fit the data at higher energy region. In this new analysis, data below about $1.9 \mathrm{GeV}$ can be fitted well. We however are not able to account for the forward peaks in the differential cross sections at $W>1.9 \mathrm{GeV}$. This might suggest a need for an incorporation of Regge-type processes at higher energy region.

\section{Nucleon resonances}

The nucleon resonance is identified by a pole of the scattering amplitude. We extract the resonance properties from the scattering amplitude obtained from the DCC model by analytic continuation. In practice, the analytic continuation can be done by choosing appropriate path of momentum integration 


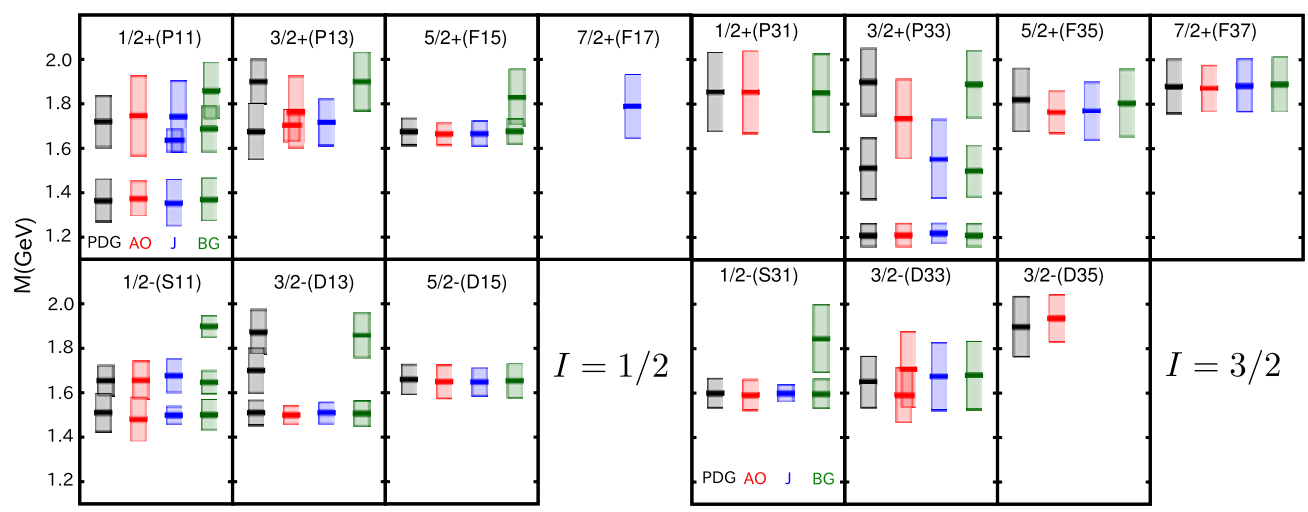

Figure 2. $N^{*}$ spectrum with the isospin $I=1 / 2$ (left) and $I=3 / 2$ (right) determined by ANL-Osaka (AO) collaboration. For each $N^{*}$ state, $\operatorname{Re}\left(M_{R}\right)$ together with the $\operatorname{Re}\left(M_{R}\right) \pm \operatorname{Im}\left(M_{R}\right)$ band is plotted. The results are compared with 4- and 3-star states listed by the PDG [5] as well as the results from Jülich (J) (model A in Ref. [6]) and Bonn-Gatchina (BG) [7] groups. The spin and parity of states are denoted as $J^{P}$ with $P= \pm$ and the associated $\pi N$ partial waves are given in parentheses.

in solving coupled channel equation. As the energy approaches a resonance position in the complex $W$ plane, the total meson-baryon scattering amplitudes $\mathcal{F}$ can be written as

$$
\mathcal{F}_{\beta, \alpha}\left(k_{\beta}^{R}, k_{\alpha}^{R}, W\right)=-\frac{R_{\beta, \alpha}\left(M_{R}\right)}{W-M_{R}}+B_{\beta, \alpha}\left(M_{R}\right)+\mathcal{O}\left(W-M_{R}\right) .
$$

The resonance complex mass $M_{R}$ is defined as a pole position of the meson-baryon amplitude on the complex $W$ plane. All of these poles are on the unphysical sheet of the $\pi N$ channel, but could be on either "unphysical" or "physical" sheets of other channels. The residue of the scattering amplitude $R_{\beta, \alpha}\left(M_{R}\right)$, which depends on complex "on-shell" momentum $k_{\alpha}^{R}$ in DCC model, represents coupling constant $g_{N^{*} \alpha}$ of the resonance to the meson-baryon channel

$$
R_{\beta, \alpha}\left(M_{R}\right)=g_{N^{*} \beta} g_{N^{*} \alpha} .
$$

The electromagnetic helicity amplitude $A_{\lambda}$ is obtained from $g_{N^{*} \alpha}$ of the meson photoproduction amplitude.

In Fig. 2, we compare our (ANL-Osaka) [4] values (AO, 2nd column) of the $N^{*}$ spectrum with those from PDG [5] (PDG, first column) and the analyses by the Jülich [6] (J, 3rd column) and the Bonn-Gatchina [7] (BG, 4th column) groups. Here we only list the three- and four-star $N^{*}$ of PDG. Most of their widths are less than $400 \mathrm{MeV}$ that is the limit set in our search. We see that we agree well with PDG for the $N^{*}$ resonances below $2 \mathrm{GeV}$ except (a) we do not have the second and third states in $3 / 2^{-}\left(D_{13}\right)$, (b) we do not have a third state in $3 / 2^{+}\left(P_{33}\right)$, and (c) we have a second state in $3 / 2^{-}\left(D_{33}\right)$. The $N^{*}$ masses extracted from our analysis, PDG, Jülich analysis, and Bonn-Gatchina analysis agree well only in the low-mass region. In the higher mass region the differences among four results are rather large. The differences in the analysis methods and/or the data included in each analysis could lead to large disagreements. It could be attributed to the fact that in the high $W \gtrsim 1.7 \mathrm{GeV}$ region, the available data of $\pi N$ and $\gamma N$ reactions are far from complete to determine the partial-wave-amplitudes model independently. It is necessary to clarify the situation.

The extracted residues of $\pi N \rightarrow \pi N$ partial-wave amplitudes are compared with Bonn-Gatchina analysis and Jülich analysis in Fig. 3. Except for the well-established $\Delta(1232)$ resonance, the three analyses agree only qualitatively even for the cases that their pole positions are close. A similar situation is also found in comparing the extracted $N^{*} \rightarrow \gamma N$ helicity amplitudes with those from the 

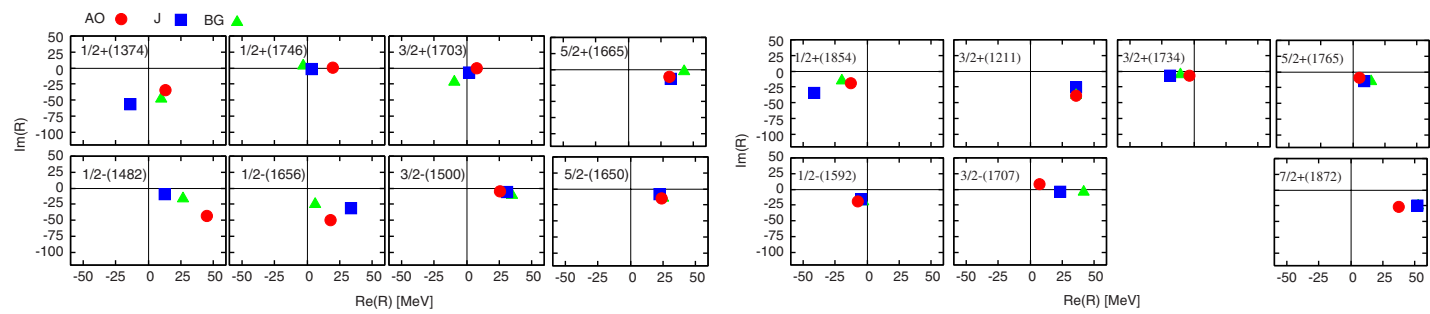

Figure 3. Residue of the $\pi N$ elastic scattering amplitudes for $I=1 / 2$ (left) and $I=3 / 2$ (right) at resonance pole.

Bonn-Gatchina analysis. This is perhaps attributable to the fact that the residues are more sensitive to the model or parametrization used in the analyses.

The DCC model developed in this work can be applied to the electron-induced meson production reactions. This analysis is a key to understanding the quark-gluon substructure of the $N^{*}$ states though the $Q^{2}$ dependence of $N-N^{*}$ electromagnetic transition form factors. The DCC model presented can be also extended straightforwardly to describe the neutrino-induced reactions in the nucleon resonance region. A first attempt to study the neutrino-induced reactions within the current DCC model has been done in Ref. [8].

To improve our analysis in the higher mass region, we need to include the data of $\pi N, \gamma N \rightarrow \pi \pi N$ reactions that dominate the $\pi N$ and $\gamma N$ reactions at $W \gtrsim 1.6 \mathrm{GeV}$. We also need to extend the analysis to include the $\omega N$ channel which has significant contributions to the $\pi N$ and $\gamma N$ reaction cross sections at $W \gtrsim 1.7 \mathrm{GeV}$. Efforts in this direction are in progress.

This report is based on Ref. [4], which has been done in collaboration with H. Kamano, S. Nakamura and T.-S.H. Lee. This work was supported by JSPS KAKENHI Grant Numbers 24540273 and 25105010.

\section{References}

[1] A. Matsuyama, T. Sato and T.-S.H. Lee, Phys. Rep. 439, 193 (2007)

[2] B. Juliá-Díaz, T.-S.H. Lee, A. Matsuyama, and T. Sato, Phys. Rev. C 76, 065201 (2007)

[3] N. Suzuki, B. Juliá-Díaz, H. Kamano, T.-S.H. Lee, A. Matsuyama, and T. Sato, Phys. Rev. Lett. 104, 042302 (2010)

[4] H. Kamano, S. Nakamura, T.-S.H. Lee and T. Sato, Phys. Rev. C 88, 035209 (2013)

[5] J. Beringer et al. (Particle Data Group), Phys. Rev. D 86, 010001 (2012)

[6] D. Rönchen, M. Döring, F. Huang, H. Haberzettl, J. Haidenbauer, C. Hanhart, S. Krewald, U.-G. Meißner, and K. Nakayama, Eur. Phys. J. A 49, 44 (2013)

[7] A.V. Anisovich, R. Beck, E. Klempt, V.A. Nikonov, A.V. Sarantsev, and U. Thoma, Eur. Phys. J. A 48, 15 (2012)

[8] H. Kamano, S.X. Nakamura, T.-S.H. Lee, and T. Sato, Phys. Rev. D 86, 097503 (2012) 